\title{
Wilms' tumour propagated through the inferior vena cava into the right heart cavities
}

\author{
Guillermo Anselmi, José A. Suárez, Iván Machado, Federico Moleiro, and \\ Pablo Blanco \\ From the Sections of Pediatric Cardiology and Hemodynamics, Department of Cardiology, \\ University Hospital, and the Institute of Pathology and Laboratory of Experimental \\ Cardiology, Ciudad Universitaria, Caracas, Venezuela
}

$A$ report is made of a rare case of Wilms' tumour which simulated clinically a syndrome of 'right heart failure', as found in some cases of Ebstein's disease. The clinical, radiological, and electrocardiographic study led to the suspicion of this type of malformation. The cine-angiographic study revealed two important facts: the impossibility of approaching the inferior vena cava with opaque material injected into the azygos vein and a filling defect of the right atrium. The post-mortem study revealed the presence of a Wilms' tumour of the right kidney which extended into the inferior vєna cava and into the right atrium without producing metastases. This seems to be the first case of this peculiar course of a Wilm's tumour which has grown into the right atrium, resulting in this bizarre clinical picture.

The present report refers to an unusual case of Wilms' tumour which originated in the right kidney and through the renal vein, invaded the inferior vena cava, and ascended into the right atrium, without producing metastases. This gave rise to a peculiar heart condition, exhibiting clinical similarities with some features of Ebstein's malformations of the tricuspid valve.

\section{Case report}

A 4-year-old boy was admitted to the Department of Pediatrics of the University Hospital because of enlargement of the abdomen, oedema of the lower extremities, oliguria, and vesical tenesmus. He was the product of a normal pregnancy and full-term delivery. The past medical history was unremarkable. He had ascites 4 months before admission and oedema of the lower limbs 2 months before.

Physical examination disclosed a slightly jaundiced patient. His height was $102 \mathrm{~cm}$.; he weighed $17.5 \mathrm{~kg}$. He had ascites and moderate oedema of the legs. The jugular veins were distended; a prominent ' $a$ ' wave was a striking feature. A left parasternal impulse lift was palpable. Auscultation of the heart disclosed a soft regurgitant systolic murmur (2/6) over the tricuspid area which increased with inspiration. A 4th sound was audible over the apex, and a fixed and closely split second sound at the pulmonary area. The liver was $13 \mathrm{~cm}$. below the costal margin.

The electrocardiogram showed peaked $\mathbf{P}$ waves measuring $5.5 \mathrm{~mm}$. voltage in lead II, suggesting systolic overloading of the right atrium.
The $x$-ray films (Fig. IA, B) revealed conspicuous right atrial enlargement.

At cardiac catheterization a No. $7 \mathrm{~F}$ Lehman catheter was inserted into the right saphenous vein. Several attempts to advance the catheter into the inferior vena cava were unsuccessful. The catheter was withdrawn into a lower position where the radiopaque substance was injected. A cine-angiocardiogram (Fig. IC, D) showed absence of the inferior vena cava, filling of the azygos and hemiazygos veins, with multiple anastomoses between both vessels; filling of the superior vena cava and the right atrium which was conspicuously enlarged. The tricuspid valve was displaced upward and to the left. At first a filling defect in the right atrium was not recognized. The venous pressure was $20 \mathrm{~mm}$. $\mathrm{Hg}$ and the arterial $\mathrm{O}_{2}$ saturation 99 per cent.

Laboratory examinations revealed that urinalysis, bilirubin, total and fractionate proteins were normal.

The patient improved with treatment for heart failure and was discharged from hospital.

One month later he was readmitted with vomiting and dyspnoea; his liver again was very enlarged, the heart rate was $160 / \mathrm{min}$.; 4 hours later the patient died in cardiac arrest after resuscitation procedures had proved unsuccessful.

Necropsy The most important finding was a tumour of the right kidney, which deformed the organ; it measured $9 \times 6 \times 5.5 \mathrm{~cm}$; it had an irregular surface partly smooth and partly dented. It did not infiltrate the perirenal tissue. On section there was a whitish tissue which replaced 80 per cent of the right kidney, and invaded the right 


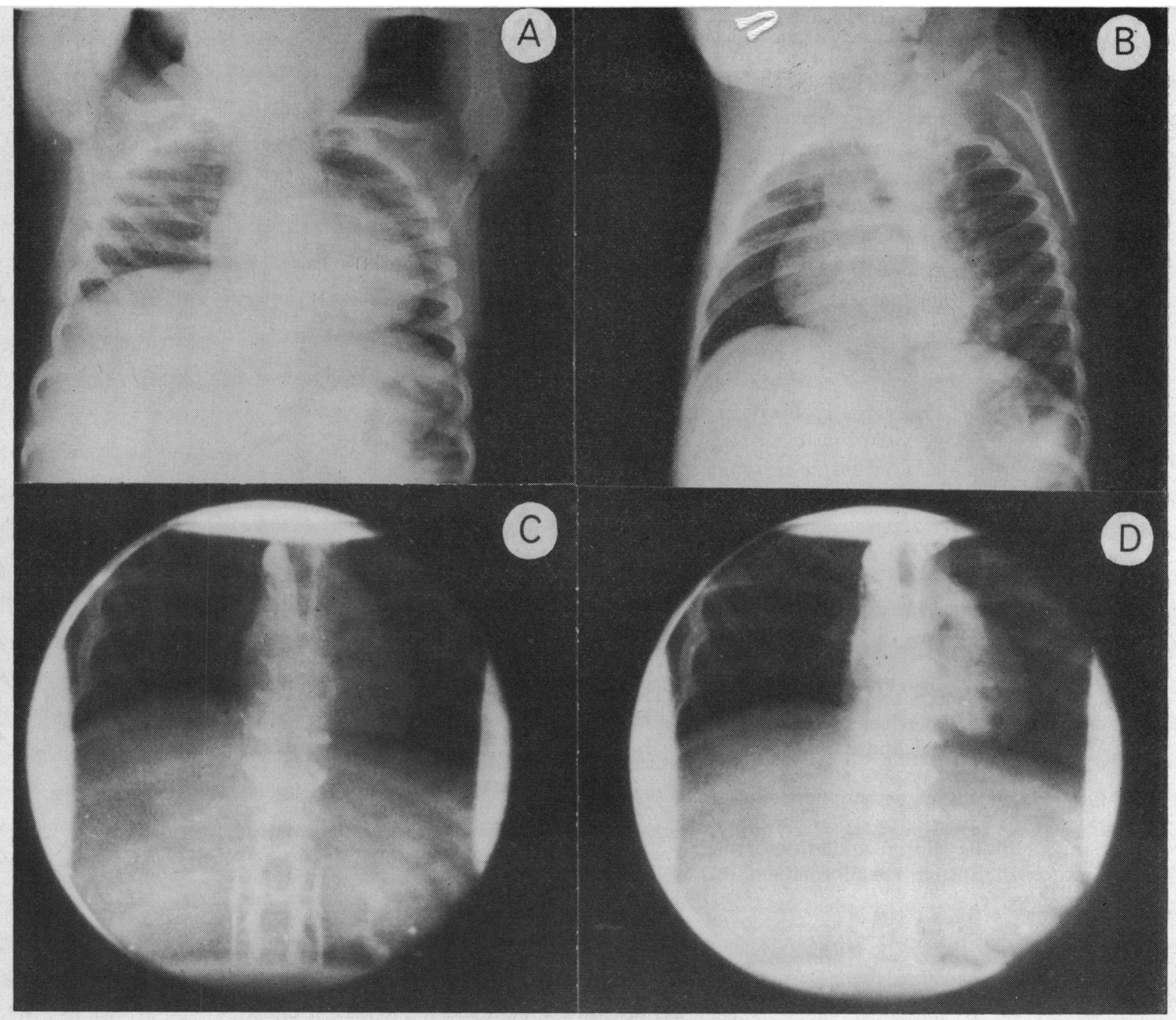

FIG. I X-ray, top: frontal $(A)$ and left oblique $(B)$ positions showing important enlargement of the right atrial appendage. Bottom: Cine-angiocardiographic study. $(C)$ The filling of the superior vena cava through the azygos vein and the anastomoses between the azygos and hemiazygos veins. (D) A filling defect in the right atrium and displacement of the tricuspid valve are apparent.

renal vein, distending and obliterating it. It extended into the inferior vena cava (Fig. 2A) and it entered the right atrium which it filled. Furthermore, it bulged into the right ventricle at the level of the atrioventricular valve (Fig. 2C); no signs of invasion of the wall of the renal vein or inferior vena cava were observed. There were no metastases.

The heart showed moderate dilatation and hypertrophy of the right ventricle and a great dilatation of the right atrium (Fig. $2 B, C$ ); the left cavities were normal. The liver was enlarged and congested; the remaining organs were unremarkable.
The microscopical study of the renal tumour (Fig. 2D), showed a structure formed by embryonic connective tissue, clearly of tumoral aspect; in the centre of it there were tubular irregular formations, a small lumen, covered with columnar epithelium of limited cytoplasm and of irregular nucleus situated at different levels: some of these tubules did not have lumina and appeared as reddish spots similar to metanephric tubules of the renal blastema. The tumour was formed mostly by embryonic connective tissue, areas of myxoid or adipose tissue and disseminated smooth muscle fibres. The periphery showed a thin band of collagen tissue forming a capsule around the tumour. 

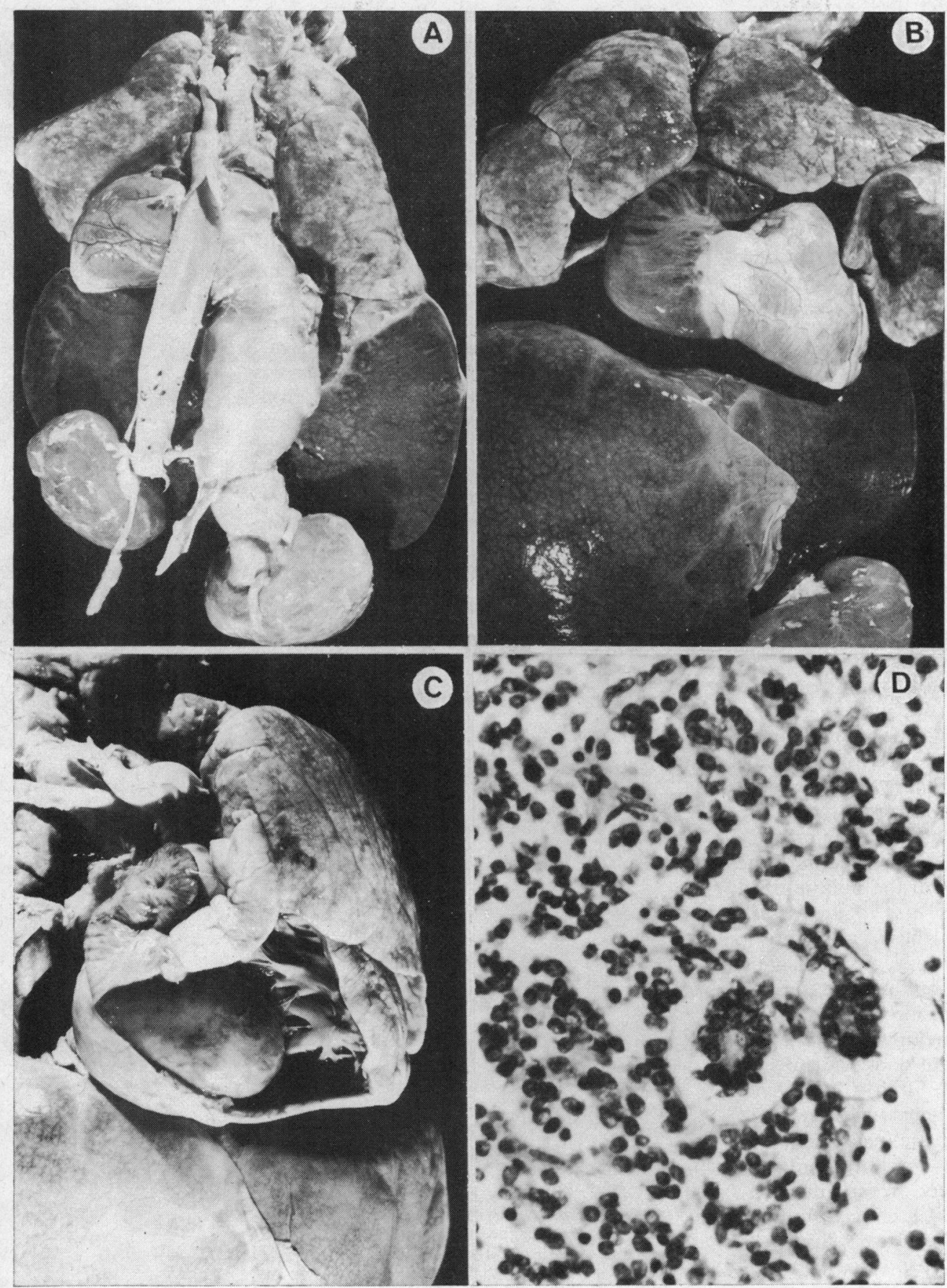

FIG. 2 Anatomical specimen and histology. (A) Posterior view of the thoracic and abdominal organs. Note the right kidney enlarged, with the tumour and a grossly distended inferior vena cava. (B) Note the enlargement of the right atrium and atrial appendage. Congestive hepatomegaly. $(C)$ Right atrium and ventricle opened: the encapsulated tumour protrudes through the atrium obstructing the tricuspid orifice. (D) Microscopically the tumour presented undifferentiated tumoral tissue in the middle of which two metanephric tubular-like structures can be seen. 
These features are consistent with the diagnosis of a nephroblastoma (Wilms' tumour). The encapsulated growth which entered the renal vein, the inferior vena cava, and the right atrium, and the absence of metastases were remarkable.

\section{Discussion}

According to Schaffer (1960), Wilms' tumour is the second most common of the abdominal tumours in children. It is an embryonic tumour which originates from the primitive mesenchyma from which the kidney is formed. At birth it may present a certain amount of growth, and gives rise to symptoms almost always before 5 years of age. When the tumours become palpable they are already of considerable size, and have usually given rise to metastases. The prognosis of the tumour and the surgical results are poor. At the beginning the tumour is encapsulated; but it soon metastasizes, particularly to the brain, the liver, and the lungs. It is rare for the nephroblastoma to make progress through the renal vein and inferior vena cava into the right heart cavities, as shown by Macgregor (1960) and in our case. To date we have not found a description of the clinical findings and the cardiovascular signs of similar cases. The encapsulated nephroblastoma progressed up into the right heart cavities via the inferior vena cava, which it obstructed: an uncommon fact, since obstructions of the inferior vena cava have been reported mainly in adults, and according to Deutsch et al. (1968) they are caused by leiomyosarcomas and other primary venous tumours. When the obstruction of the inferior vena cava occurs in the middle third of the vessel (López et al., 1968; Missal, Robinson, and Tatum, 1965), a nephrotic syndrome frequently develops, a complication that was not present in our patient, despite the obstruction of the inferior vena cava from the level of the renal veins upwards. The obstruction of the upper third of the inferior vena cava produces the Budd-Chiari syndrome characterized by hepatosplenomegaly, ascites, jaundice, and hepatic insufficiency.

Our patient presented with hepatomegaly and ascites but not jaundice, hepatic insufficiency, or splenomegaly. The clinical findings were suggestive of 'right heart failure', as found in the final phases of some cases of $\mathrm{Eb}$ stein's malformation of the tricuspid valve, when there is significant stenosis of the tricuspid orifice, with great dilatation of the right atrium.

Partial absence of the inferior vena cava is relatively frequent in association with certain congenital malformations, and they are commonly suspected when the catheter inserted in the femoral vein cannot be advanced. An injection of contrast medium in a low position will show the filling of the azygos vein emptying into the superior vena cava, or of the hemiazygos vein with its accessory vein, emptying into the persistent left superior vena cava. In our patient, the venous angiography showed 'absence of the inferior vena cava', the contrast material ascended via the azygos vein into the superior vena cava, and from there it entered the right atrium; this led us to believe that there was partial absence of the inferior vena cava in association with $\mathrm{Eb}$ stein's malformation of the tricuspid valve. Assuming that the interpretation of the filling defect of the right atrium in the cine-angiocardiogram was suggestive of an atrial tumour, it is still difficult to explain the obstruction beneath the inferior vena cava and to relate it to the same cause. As far as we have been able to see in the literature, we have not found a case of Wilms' tumour with this peculiar behaviour. There are reported cases of primary tumours of the inferior vena cava which progress into the right atrium, though to date only one, a case of leiomyosarcoma in an adult, has been diagnosed by venous angiography (Deutsch et al., I968).

Deutsch, V., Fraenkel, O., Frand, U., and Hulu, N. (1968). Leiomyosarcoma of the inferior vena cava propagating into the right atrium. British Heart fournal, 30, $57 \mathrm{I}$.

López, J. E., Martínez, F., Gubaira, J. A., and Arocha, R. (1968). Síndrome nefrótico producido por obstrucción de la vena cava inferior. Acta Medica Venezolana, 15, 218.

Macgregor, A. R. (1960). Pathology of Infancy and Childhood. E. \& S. Livingstone, Edinburgh and London.

Missal, M. E., Robinson, J. A., and Tatum, R. W. (1965). Inferior vena cava obstruction. Clinical manifestations, diagnostic methods, and related problems. Annals of Internal Medicine, 62, 133.

Schaffer, A. J. (1960). Diseases of the Newborn. W. B. Saunders, Philadelphia and London. 